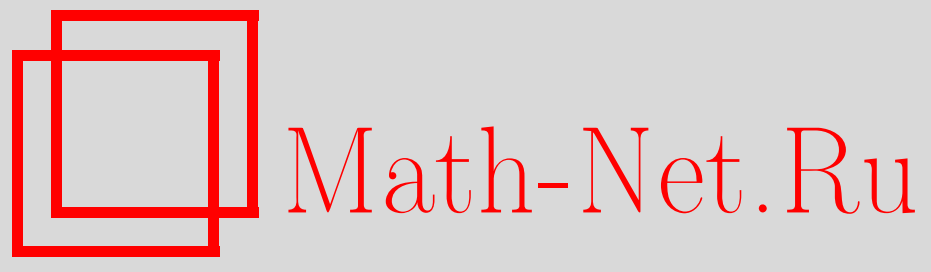

Р. Де Лео, Замечание о несвободных изометрических погружениях, УМН, 2010, том 65, выпуск 3, 197-198

DOI: https://doi.org/10.4213/rm9359

Использование Общероссийского математического портала Math-Net.Ru подразумевает, что вы прочитали и согласны с пользовательским соглашением http://www . mathnet.ru/rus/agreement

Параметры загрузки:

IP: 54.166 .219 .16

26 апреля 2023 г., $15: 36: 49$

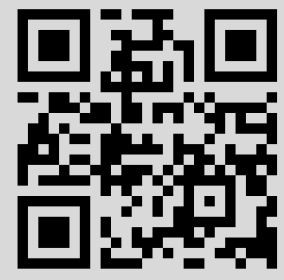




\section{Замечание о несвободных изометрических погружениях}

\section{Р. Де Лео}

Задача об изометрических погружениях впервые изучалась в выдающейся работе [1] Дж. Нэша, а затем, в значительно более общей ситуации, в работе М. Громова [2]. В этом контексте было показано, что для гладкого $m$-мерного многообразия $M$ и множества $\Gamma(M)$ всех квадратичных дифференциалов на нем индуцирующее метрику отображение $\mathscr{D}: C^{\infty}\left(M, \mathbb{R}^{q}\right) \rightarrow \Gamma(M)$, сопоставляющее каждому отображению $f: M \rightarrow \mathbb{R}^{q}$ прообраз $f^{*} e_{q}$ евклидовой метрики $e_{q}$ на $\mathbb{R}^{q}$, открыто, будучи ограничено на множество свободных отображений (мы снабжаем все функциональные пространства топологией Уитни). Напомним, что отображение $f=\left(f^{i}\right): M \rightarrow \mathbb{R}^{q}$, $i=1, \ldots, q$, свободно, если в произвольной карте $\left(x^{\alpha}\right)$ многообразия $M, \alpha=1, \ldots, m$, набор из $q_{m}:=m(m+3) / 2$ векторов $\left\{\partial_{\alpha} f, \partial_{\alpha \beta} f\right\}$ линейно независим в каждой точке.

Множество $F\left(M, \mathbb{R}^{q}\right)$ всех свободных отображений есть открытое подмножество в $C^{\infty}\left(M, \mathbb{R}^{q}\right)$, известно, что оно пусто при $q<q_{m}$ и всюду плотно при $q \geqslant q_{m}+m$. Поэтому отображение $\mathscr{D}$ открыто над некоторым плотным множеством при $q \geqslant q_{m}+m$ и открыто по крайней мере над непустым открытым множеством (тогда $\mathscr{D}$ называется $w$-открытым $)$ в тех случаях, когда $q_{m} \leqslant q<q_{m}+m$ и $F\left(M, \mathbb{R}^{q}\right)$ непусто. Очень мало известно о том, в каких случаях операторы $\mathscr{D}$ открыты для меньших значений $q$, когда свободных отображений появиться не может по размерностным соображениям, кроме очевидного факта, что они не могут быть открытыми при $q \leqslant q_{m}-m$.

Гипотеза М. Громова $\left[2 ;\right.$ гл. $2, \S 3$, п. 8 , случай $\left.\left(\mathrm{E}^{\prime}\right)\right]$ утверждает, что операторы $\mathscr{D}$ открыты над всюду плотным множеством для всех $q \geqslant q_{m}-\sqrt{m / 2}$. Мы изучаем задачу, когда операторы $\mathscr{D} w$-открыты, и концентрируемся на случае $M=\mathbb{R}^{m}$. Обозначим через $\mathscr{D}_{m, q}$ оператор $\mathscr{D}$, действующий на $C^{\infty}\left(\mathbb{R}^{m}, \mathbb{R}^{q}\right)$. Так как $F\left(\mathbb{R}^{m}, \mathbb{R}^{q}\right)$ при $q \geqslant q_{m}$ непусто (см. пример 2 ниже), наша задача нетривиальна только при $q \leqslant q_{m}-1$.

В [3] показано, что $\mathscr{D}_{2,4} w$-открыт. Мы распространяем этот результат на $\mathscr{D}_{m, q_{m}-1}$, основываясь по существу на следующем хорошо известном результате из [4].

Teорема DH. Пусть $M$ - открытое многообразие, $\lambda$ и $\xi$ - гладкая функиия $и$ гладкое векторное поле на нем. Тогда оператор $X(f)=\xi(f)+\lambda f$ на $C^{\infty}(M)$ сюрвективен тогда и только тогда, когда $\xi$ допускает глобальную трансверсаль, т.е. вложенную гиперповерхность, которая пересекает каждую его интегральную траекторию в единственной точке.

Пусть $q=q_{m}-1$. Наша цель - найти открытое множество $\mathscr{A}_{m, q} \subset C^{\infty}\left(\mathbb{R}^{m}, \mathbb{R}^{q}\right)$ такое, что если $f_{0} \in \mathscr{A}_{m, q}$ и $g_{0}=\mathscr{D}_{m, q}\left(f_{0}\right)$, то уравнение $\mathscr{D}_{m, q}(f)=g$ имеет решение для любого $g$, достаточно близкого к $g_{0}$ в $\Gamma\left(\mathbb{R}^{m}\right)$. Напомним, что по общей фундаментальной теореме Громова (см. [2; гл. $2, \S 3$, п. 2]) существование решения уравнения $\mathscr{D}_{m, q}(f)=g$ обеспечивается существованием решений его линеаризованного варианта, который в свою очередь эквивалентен (см., например, [2; гл. $2, \S 3$, п. 8 , случай $\left.\left.\left(\mathrm{E}^{\prime}\right)\right]\right)$ алгебраической системе:

$$
\left\{\begin{array}{l}
\delta_{i j} \partial_{\alpha} f^{i} \delta f^{j}=h_{\alpha} \\
\delta_{i j} \partial_{\alpha \beta} f^{i} \delta f^{j}=\left(\partial_{\alpha} h_{\beta}+\partial_{\beta} h_{\alpha}-\delta g_{\alpha \beta}\right) / 2,
\end{array}\right.
$$

где $\delta f^{i}$ - это $q$ неизвестных, $\delta g_{\alpha \beta}$ - это $m(m+1) / 2$ данных функций, а $h_{\alpha}$ - это $m$ произвольных функций. Следовательно, для наших целей достаточно показать, что для некоторого непустого открытого множества в $C^{\infty}\left(\mathbb{R}^{m}, \mathbb{R}^{q}\right)$ всегда можно выбрать $h_{\alpha}$ так, чтобы система (1) имела решение.

Teорема DH. Операторы $\mathscr{D}_{m, q}$ являются $w$-открытыми при $q \geqslant q_{m}-1$.

ДоКАЗАТЕЛЬСтво. Коэффициенты в (1) совпадают с компонентами наших $q_{m}$ векторных полей $\left\{\partial_{\alpha} f, \partial_{\alpha \beta} f\right\}$, поэтому найдутся не обращающиеся тождественно в нуль функции $\lambda^{\alpha}$ и $\lambda^{\alpha \beta}=\lambda^{\beta \alpha}$ такие, что имеет место тождество $\lambda^{\alpha} \partial_{\alpha} f+\lambda^{\alpha \beta} \partial_{\alpha \beta} f=0$. 
Система (1) имеет решение, если и только если $2 \lambda^{\alpha} h_{\alpha}+\lambda^{\alpha \beta}\left(\partial_{\alpha} h_{\beta}+\partial_{\beta} h_{\alpha}-\delta g_{\alpha \beta}\right)=0$. Перепишем это в виде $X^{\alpha} h_{\alpha}=\phi$, где $\phi=\lambda^{\alpha \beta} \delta g_{\alpha \beta}$ и $X^{\alpha}=\xi_{\alpha}+2 \lambda^{\alpha}$ с $\xi_{\alpha}=\lambda^{\alpha \beta} \partial_{\beta}$.

Теперь пусть $\mathscr{A}_{m, q} \subset C^{\infty}\left(\mathbb{R}^{m}, \mathbb{R}^{q}\right)$ - открытое множество погружений $f$, удовлетворяющее следующим открытым свойствам: 1$)$ матрица $D^{2} f$ размера $q_{m} \times\left(q_{m}-1\right)$ имеет полный ранг в каждой точке; 2$)$ найдется индекс $\alpha_{0}$, для которого функции $\lambda^{\alpha_{0} \beta}$ не обращаются в нуль все одновременно. Тогда, если положить $h_{\beta}=\lambda^{\alpha_{0} \beta} h$, $\beta=1, \ldots, m$, для некоторой неизвестной функции $h$, то уравнение $X^{\alpha} h_{\alpha}=\phi$ превращается в $Y h=\phi^{\prime}$, где $Y=\zeta+\lambda^{\prime}$ для некоторого векторного поля $\zeta$ и функции $\lambda^{\prime}$. Прямое вычисление показывает, что компонента $\alpha_{0}$ поля $\zeta$ равна $\left(\lambda^{\alpha_{0} 1}\right)^{2}+\cdots+\left(\lambda^{\alpha_{0} m}\right)^{2}$ и, следовательно, никогда не обращается в нуль по предположению. В частности, это означает, что любая поверхность $x^{\alpha_{0}}=$ const является глобальной трансверсалью для $\zeta$, и, следовательно, по теореме DH, $Y$ - сюръективный дифференциальный оператор первого порядка. Значит, для любой функции из $\mathscr{A}_{m, q}$ всегда можно выбрать $h_{\alpha}$ в определении функций $\delta g_{\alpha \beta}$ так, чтобы выполнялось условие совместности $X^{\alpha} h_{\alpha}=\phi$. Примеры 1 и 2 показывают, что эти множества непусты.

Пример 1. Рассмотрим произвольную пару $(g, h)$ свободных отображений из $\mathbb{R}$ в $\mathbb{R}^{2}$. Тогда функция $f: \mathbb{R}^{2} \rightarrow \mathbb{R}^{4}$, заданная формулой $f(x, y)=(g(x), h(y))$, принадлежит $\mathscr{A}_{2,4} \subset C^{\infty}\left(\mathbb{R}^{2}, \mathbb{R}^{4}\right)$. Действительно, в этом случае $\partial_{x y} f=0$, так что можно выбрать $\lambda^{x}=\lambda^{y}=\lambda^{x x}=\lambda^{y y}=0, \lambda^{x y}=\lambda^{y x}=1$ и, значит, условие совместности превращается в тривиально разрешимое: $\partial_{x} h_{y}+\partial_{y} h_{x}=\delta g_{x y}$. Например, функция $f(x, y)=\left(x, e^{x}, y, e^{y}\right)$ лежит в $\mathscr{A}_{2,4}$. Заметим, что наше множество $\mathscr{A}_{2,4}$ строго больше множества, введенного в [3].

ЗАмечАниЕ. Пусть $\mathscr{D}_{q}-$ индуцирующий метрику оператор, действующий на $C^{\infty}\left(\mathbb{T}^{2}, \mathbb{R}^{q}\right)$. Случаи $q \geqslant 7$ и $q \leqslant 3$ тривиальны. Пример 1 показывает, что $\mathscr{D}_{4}$ является $w$-открытым. Действительно, множество $\mathscr{A}_{2,4}$ содержит функции, двояко периодичные, например, $f(x, y)=(\cos x, \sin x, \cos y, \sin y)$. Подмножество, состоящее из всех них, рассматриваемых как функции на $\mathbb{T}^{2}$, открыто в $C^{\infty}\left(\mathbb{T}^{2}, \mathbb{R}^{4}\right)$, и $\mathscr{D}_{4}$ очевидно является открытым отображением над ним. Отметим, что случай $q=6$ тривиален, поскольку $F\left(\mathbb{T}^{2}, \mathbb{R}^{6}\right) \neq \varnothing$, в то время как ничего нельзя сказать в случае $q=5$, поскольку до сих пор неизвестно, является ли $F\left(\mathbb{T}^{2}, \mathbb{R}^{5}\right)$ пустым.

Пример 2. Пусть $f \in F\left(\mathbb{R}^{m}, \mathbb{R}^{q_{m}}\right)$ - каноническое свободное отображение, заданное формулой $f\left(x^{1}, \ldots, x^{m}\right)=\left(x^{1}, \ldots, x^{m},\left(x^{1}\right)^{2}, x^{1} x^{2}, \ldots,\left(x^{m}\right)^{2}\right)$, и $\pi$ - произвольная проекция $\pi: \mathbb{R}^{q_{m}} \rightarrow \mathbb{R}^{q_{m}-1}$, забывающая любую из последних $(m+1) / 2$ компонент. Тогда композиция $f_{\pi}=\pi \circ f$ принадлежит $\mathscr{A}_{m, q_{m}-1}$. Действительно, матрица $D^{2} f_{\pi}$ имеет полный ранг и одна из вторых производных функции $f_{\pi}$ (скажем, $\partial_{x^{1} x^{2}} f_{\pi}$ ) тождественно нулевая, так что можно выбрать соответствующий множитель (в этом случае $\lambda^{x^{1} x^{2}}$ ) тождественно равным единице, а остальные - нулю. Например, в случае $(m, q)=(2,4)$ мы получим функции $f_{1}(x, y)=\left(x, y, x y, y^{2}\right), f_{2}(x, y)=\left(x, y, x^{2}, y^{2}\right)$ и $f_{3}(x, y)=\left(x, y, x^{2}, x y\right)$.

Автор благодарит G. D'Ambra, A. Loi и A. Bergamasco за введение в предмет и полезные обсуждения.

\section{Список литературы}

[1] J. Nash, Ann. of Math. (2), 63:1 (1956), 20-63. [2] М. Громов, Дифференциальные coomношения с частными производными, Мир, М., 1990, ISBN: 5-03-001297, 536 с. [3] G. D'Ambra, A. Loi, Geom. Dedicata, 127 (2007), 151-158. [4] J. J. Duistermaat, L. Hörmander, Acta Math., 128:1 (1972), 183-269.

P. Де Леo (R. De Leo)

University of Maryland, USA

E-mail: roberto.deleo@ca.infn.it, rdl@math.umd.edu
Представлено С. П. Новиковым Принято редколлегией 13.04.2010 